\title{
Prevalence and clinical significance of serum sodium variability in patients with acute-on-chronic liver diseases: a prospective multicenter study in China
}

\author{
Xue Mei ${ }^{1} \cdot \mathrm{Hai} \mathrm{Li}^{2,3} \cdot \mathrm{Guohong} \mathrm{Deng}^{4} \cdot$ Xianbo Wang $^{5} \cdot \mathrm{Xin} \mathrm{Zheng}^{6} \cdot$ Yan Huang $^{7} \cdot$ Jinjun Chen $^{8} \cdot$ Zhongji Meng $^{9}$. \\ Yanhang Gao ${ }^{10}$. Feng $\mathrm{Liu}^{11}$. Xiaobo $\mathrm{Lu}^{12} \cdot \mathrm{Yu} \mathrm{Shi}^{13,14,15}$. Yubao Zheng ${ }^{16}$. Huadong Yan ${ }^{17}$. Weituo Zhang ${ }^{18}$.

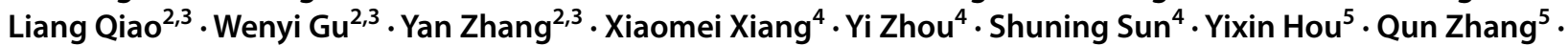

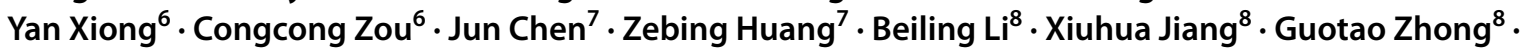

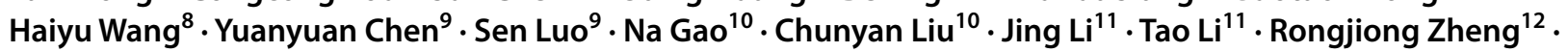 \\ Xinyi Zhou ${ }^{12} \cdot$ Haotang Ren $^{13,14,15} \cdot$ Wei Yuan ${ }^{1} \cdot$ Zhiping Qian $^{1}{ }^{10}$
}

Received: 25 August 2021 / Accepted: 22 November 2021 / Published online: 17 January 2022

(c) Asian Pacific Association for the Study of the Liver 2021

\begin{abstract}
Background No reports exist regarding the prevalence of different $\mathrm{Na}$ levels and their relationship with 90-day prognosis in hospitalized patients with acute-on-chronic liver disease (AoCLD) in China. Therefore, the benefit of hyponatremia correction in AoCLD patients remains unclear.

Methods We prospectively collected the data of 3970 patients with AoCLD from the CATCH-LIFE cohort in China. The prevalence of different Na levels $(\leq 120 ; 120-135 ; 135-145 ;>145)$ and their relationship with 90-day prognosis were analyzed. For hyponatremic patients, we measured Na levels on days 4 and 7 and compared their characteristics, based on whether hyponatremia was corrected.

Results A total of 3880 patients were involved; 712 of those developed adverse outcomes within 90 days. There were 80 (2.06\%) hypernatremic, $28(0.72 \%)$ severe hyponatremic, and $813(20.95 \%)$ mild hyponatremic patients at admission. After adjusting for all confounding factors, the risk of 90-day adverse outcomes decreased by 5\% (odds ratio [OR] 0.95; 95\% confidence interval [CI] 0.93-0.97; $p<0.001$ ), 24\% (OR 0.76; 95\% CI 0.70-0.84; $p<0.001$ ), and 42\% (OR 0.58; 95\% CI $0.49-0.70 ; p<0.001)$ as $\mathrm{Na}$ level increased by 1,5 , and $10 \mathrm{mmol} / \mathrm{L}$, respectively. Noncorrection of hyponatremia on days 4 and 7 was associated with 2.05-fold (hazard ratio [HR], 2.05; 95\% CI, 1.50-2.79; $p<0.001$ ) and 1.46-fold (HR 1.46; 95\% CI 1.05-2.02; $p=0.028$ ) higher risk of adverse outcomes.

Conclusions Hyponatremia was an independent risk factor for a poor 90-day prognosis in patients with AoCLD. Failure to correct hyponatremia in a week after admission was often associated with increased mortality. (ClinicalTrials.gov number: NCT02457637, NCT03641872).

Clinical Trial Numbers This study is registered at Shanghai www.clinicaltrials.org (NCT02457637 and NCT03641872).

Keywords Prevalence $\cdot$ Significance $\cdot$ Serum sodium $\cdot \mathrm{Na} \cdot$ Hyponatremia $\cdot$ Adverse outcome $\cdot 90$-day $\cdot$ Prognosis $\cdot$ Acuteon-chronic liver disease $\cdot$ AoCLD
\end{abstract}

Xue Mei, Hai Li, Guohong Deng, Xianbo Wang, Xin Zheng and Yan Huang contributed equally to this work.

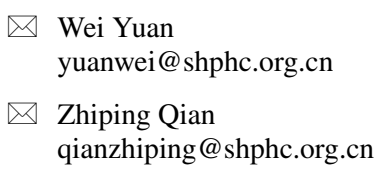

Wei Yuan

yuanwei@shphc.org.cn

$\triangle$ Zhiping Qian

qianzhiping@shphc.org.cn

Extended author information available on the last page of the article

\section{Introduction}

With the development of the global economy and the popularization of antiviral drugs, chronic hepatitis $\mathrm{B}$ and $\mathrm{C}$ have been gradually controlled; however, the incidence of fatty liver disease and drug-induced liver injury is increasing annually [1]. Chronic liver disease (CLD) remains a frequent cause of death. In 2017, 1.32 million people died directly because of liver cirrhosis, which accounts for $2-4 \%$ of 
deaths annually [2, 3]. During the coronavirus disease 2019 (COVID-19) pandemic, mortality was also significantly higher in patients with CLD than in those without CLD [4]. To improve the prognosis of cirrhotic patients, the model for end-stage liver disease (MELD) score has been used since 2002 to rank and prioritize liver transplantation (LT) candidates $[5,6]$. Since then, some researchers have found that hyponatremia was an independent prognostic factor in cirrhotic patients [7-9]. Therefore, more optimized prediction tools, such as the Model for end-stage liver disease-sodium (MELD-Na) and United Kingdom Model for end-stage liver disease (UKELD) scores, have been developed [9-11]. Most previous studies confirm that serum sodium (Na) is closely associated with the severity and complications of liver cirrhosis and affects a patient's health-related quality of life (HRQOL) and perioperative mortality after transplantation [12, 13]. Hyponatremic patients have longer hospital stays and higher hospitalization rates [14]. The subsequent mortality of patients with hyponatremia is also significantly increased, regardless of accompanying diseases [15].

An abnormal $\mathrm{Na}$ level seems to be a common electrolyte disorder in clinical practice, but it often indicates a risk of other complications. However, there was insufficient information regarding the prevalence of different $\mathrm{Na}$ levels and their relationship with 90-day prognosis in hospitalized patients with acute-on-chronic liver disease (AoCLD) in areas with a high prevalence of hepatitis B virus (HBV) in China [16]. The benefit of hyponatremia correction at 4-7 days after admission in patients with AoCLD remains unclear. Therefore, this study aimed to describe the prevalence and clinical significance of $\mathrm{Na}$ variability in this population.

\section{Methods}

\section{Patients}

In total, 3970 patients with AoCLD were included in this study from the CATCH-LIFE cohort [16-18], which was a multicenter prospective cohort study conducted by the Chinese Chronic Liver Failure (CLIF) Federation (comprising 15 tertiary hospitals in a high HBV epidemic area) conducted from 2015 to 2016 and from 2018 to 2019. The ethics committee of Ren Ji Hospital, School of Medicine, Shanghai Jiaotong University (Shanghai, China) approved the study and registered it at Shanghai www.clinicaltrials. org (NCT02457637, NCT03641872). The study was performed in accordance with the standards of the Declaration of Helsinki. Written informed consent was obtained from all patients. All authors have access to the study data and reviewed and approved the final manuscript.

\section{Related event definitions}

AoCLD is a nonmalignant CLD with acute decompensation (AD) or acute liver injury (ALI). CLD is considered cirrhotic or noncirrhotic liver disease with a history of liver dysfunction lasting $>6$ months. Cirrhosis was diagnosed based on findings of computed tomography/magnetic resonance imaging (CT/MRI) scans and laboratory tests, clinical symptoms, and history of liver disease. A diagnosis of AD requires individuals to have at least one acute decompensation event, which includes gastrointestinal hemorrhage, hepatic encephalopathy, overt ascites, bacterial infection (e.g., spontaneous peritonitis and pneumonia), or jaundice [i.e., total bilirubin (TBIL) $>5 \mathrm{mg} / \mathrm{dL}$ ] within 1 month before enrollment. ALI was defined as alanine aminotransferase or aspartate aminotransferase levels three times more than the upper limit of the normal range (ULN) (but $<40$ IU/L) or a total bilirubin level two times more than the ULN (but $<1 \mathrm{mg} / \mathrm{dL}$ ) within a week. The definition of 90-day adverse outcomes or poor prognosis was death or LT.

Based on the definition of hyponatremia [19-21], all patients were classified into four groups as follows: severe hyponatremia ( $\mathrm{Na}$ level, $\leq 120 \mathrm{mmol} / \mathrm{L}$ ), mild hyponatremia (Na level, $>120$ and $<135 \mathrm{mmol} / \mathrm{L}$ ), normal serum sodium ( $\mathrm{Na}$ level, $\geq 135$ and $\leq 145 \mathrm{mmol} / \mathrm{L}$ ), and hypernatremia ( $\mathrm{Na}$ level $>145 \mathrm{mmol} / \mathrm{L}$ ) groups. The exclusion criteria were as follows: (1) age $<15$ years or $>80$ years, (2) pregnancy, (3) severe chronic extrahepatic disease, and (4) hepatocellular carcinoma or other liver malignancies before or during admission, and extrahepatic malignancies.

\section{Data collection}

Data pertaining to demographics, medical history, biochemical indicators, and imaging data were prospectively recorded at each center. After admission, all patients were followed up for 90 days, and the outcomes were recorded (endpoint events included LT or death).

\section{Statistical analysis}

Data were summarized using the appropriate descriptive statistics (i.e., median and interquartile range for continuous variables and frequency and percentage for categorical parameters). The Mann-Whitney $U$ test was used for nonparametric pairwise comparisons, and the Chi-square test or Fisher's exact test was used for categorical variables. For more than three variables, non-normal continuous variables were analyzed using the Kruskal-Wallis test.

We estimated the potential confounding factors that impact the prognosis of AoCLD patients by univariate and 
subgroup analyses, which showed a statistically significant interaction $(p<0.05)$. When Na level was used as a continuous variable and a categorical variable, multiple covariates (i.e., potential confounding factors) were gradually introduced to analyze the influence of unadjusted and multivariate-adjusted $\mathrm{Na}$ level on 90-day adverse outcomes using the backward stepwise (likelihood ratio) method of binary logistic regression. Furthermore, a generalized additive model and smooth curve fitting were used to describe the relationship between Na level and 90-day adverse outcomes. Survival curves were estimated using the Kaplan-Meier method and compared using the log-rank test. The main objective of this study was to assess the effect of $\mathrm{Na}$ level on the risk of 90-day adverse outcomes.

All statistical tests were two-tailed, and bilateral $\alpha$ of $<0.05$ was statistically significant. SPSS 25 (IBM, Seattle, WA, USA) and R version 4.0.4 software (http://www.rproject.org) were used for statistical analysis and plots.

\section{Results}

\section{Characteristics of the study population}

Finally, 3880 patients were analyzed in this study according to the inclusion and exclusion criteria (Fig. 1). We determined not more than 5\% missing data for each variable used on admission (Supplementary Fig. 1). The prevalence of different $\mathrm{Na}$ levels (i.e., hyponatremia, normonatremia, hypernatremia) in patients with AoCLD and their characteristics are summarized in Table 1. The incidence of alcoholic liver disease and chronic hepatitis B were significantly different between the $\mathrm{Na}$ level groups $(p<0.05)$.

The lower the Na level, the higher the proportion of liver cirrhosis and the worse the liver function score, including MELD, sequential organ failure assessment (SOFA), Child-Pugh, and other related scores $(p<0.05)$. At admission, patients in the hyponatremic groups had a higher frequency of $\mathrm{AD}$ events (e.g., ascites, hepatic encephalopathy, infection, and jaundice, but not gastrointestinal bleeding; $p<0.05)$. Moreover, TBIL, international normalized ratio (INR), serum creatinine (CR) level, blood urea nitrogen, and white blood cell (WBC) counts were also higher in the hyponatremic groups $(p<0.05)$ (Table 1; Supplementary Fig. 2).

\section{Ninety-day adverse outcomes}

Among the 3880 patients, 712 (18.35\%) had 90-day adverse outcomes (498 deaths and 214 LT). The severe hyponatremia group had the highest incidence of adverse outcomes
$(50.0 \%, p<0.05)$ and LT-free mortality $(48.2 \%, p<0.05)$ (Table 1; Supplementary Fig. 3a).

\section{Relationship between hyponatremia and 90-day adverse outcomes}

Interactions between $\mathrm{Na}$ level and 90-day adverse outcomes were observed in subgroups with different TBIL levels, INR, CR levels, age, and incidence of cirrhosis, ascites, infection, and hepatic encephalopathy $(p<0.05)$, but not for sex and etiology (Supplementary Fig. 3b). Among patients with hepatitis $\mathrm{B}$, regardless of receiving nucleotide/nucleoside analogs (NUCs) before admission, $\mathrm{Na}$ level remained an independent risk factor for 90-day adverse outcomes. We constructed four models (i.e., 1 unadjusted and 3 adjusted) to evaluate the relationship between $\mathrm{Na}$ level and 90-day adverse outcomes. Univariate and multivariate analyses showed that $\mathrm{Na}$ levels were inversely associated with the incidence of 90-day adverse outcomes (for trend, $p<0.001$ ) (Table 2). After adjusting for all confounding factors that may affect the outcomes, the severe hyponatremia group had the highest risk of 90-day adverse outcomes (odds ratio [OR] 2.36; $95 \%$ confidence interval $[\mathrm{CI}] 0.98-5.67 ; p=0.056)$. Therefore, hyponatremia was an independent risk factor for 90-day adverse outcomes in patients with AoCLD. When Na level was analyzed as a continuous variable, the risk of 90 -day adverse outcomes decreased by $5 \%$ (OR $0.95 ; 95 \%$ CI 0.93-0.97; $p<0.001$ ), 24\% (OR, 0.76; 95\% CI 0.70-0.84; $p<0.001$ ), and $42 \%$ (OR 0.58 ; 95\% CI $0.49-0.70$; $p<0.001$ ) for $\mathrm{Na}$ level increasing by 1,5 , and $10 \mathrm{mmol} / \mathrm{L}$, respectively, after adjusting for all confounding factors. Similar results were obtained for the cirrhosis subgroup (Table 3). In the noncirrhotic population, hyponatremia increased the risk of 90-day adverse outcomes; however, no significant difference existed after adjusting for all confounding factors (Table 4). Finally, we found that the results were consistent with the original data, based on sensitivity analysis of multiple imputation missing values (Supplementary Tables 1-3).

The generalized additive model showed that, when the $\mathrm{Na}$ level was $<135 \mathrm{mmol} / \mathrm{L}$, the relationship between the $\mathrm{Na}$ level and the incidence of 90-day adverse outcomes was approximately linear and negatively correlated, regardless of the correction of the confounding factors (Fig. 2a,b). However, when the $\mathrm{Na}$ level was $>135 \mathrm{mmol} / \mathrm{L}$, the risk of 90-day adverse outcomes did not significantly increase. Therefore, a Na level of $135 \mathrm{mmol} / \mathrm{L}$ could be used as the cut-off value. The risk of 90-day adverse outcomes in patients with hyponatremia ( $\mathrm{Na}$ level $<135 \mathrm{mmol} / \mathrm{L}$ ) was significantly higher than that in patients without hyponatremia (Na level $\geq 135 \mathrm{mmol} / \mathrm{L}$ ) (hazard ratio [HR], 3.01; 
3655 patients with eirrhosis or other chronic liver disease hospitalized for acute decompensations and lor acute liver injury(From January 2015 to December 2016)

1055 Exeluded
36 Ageclsy or age $>80 y$
22 Pregant
591 Found liver malignancy or
other organs malignancies
107 Had severe chronic
extra-hepatic discases
21 Received immunosuppressive
drugs for reasons other than
chronic liver diseases
73 Withdrew informed consent
205 Others

1849 patients with eirrhosis or other chronic liver disease hospitalized for acute decompensations and lor acute liver injury(From September 2018 to January 2019)

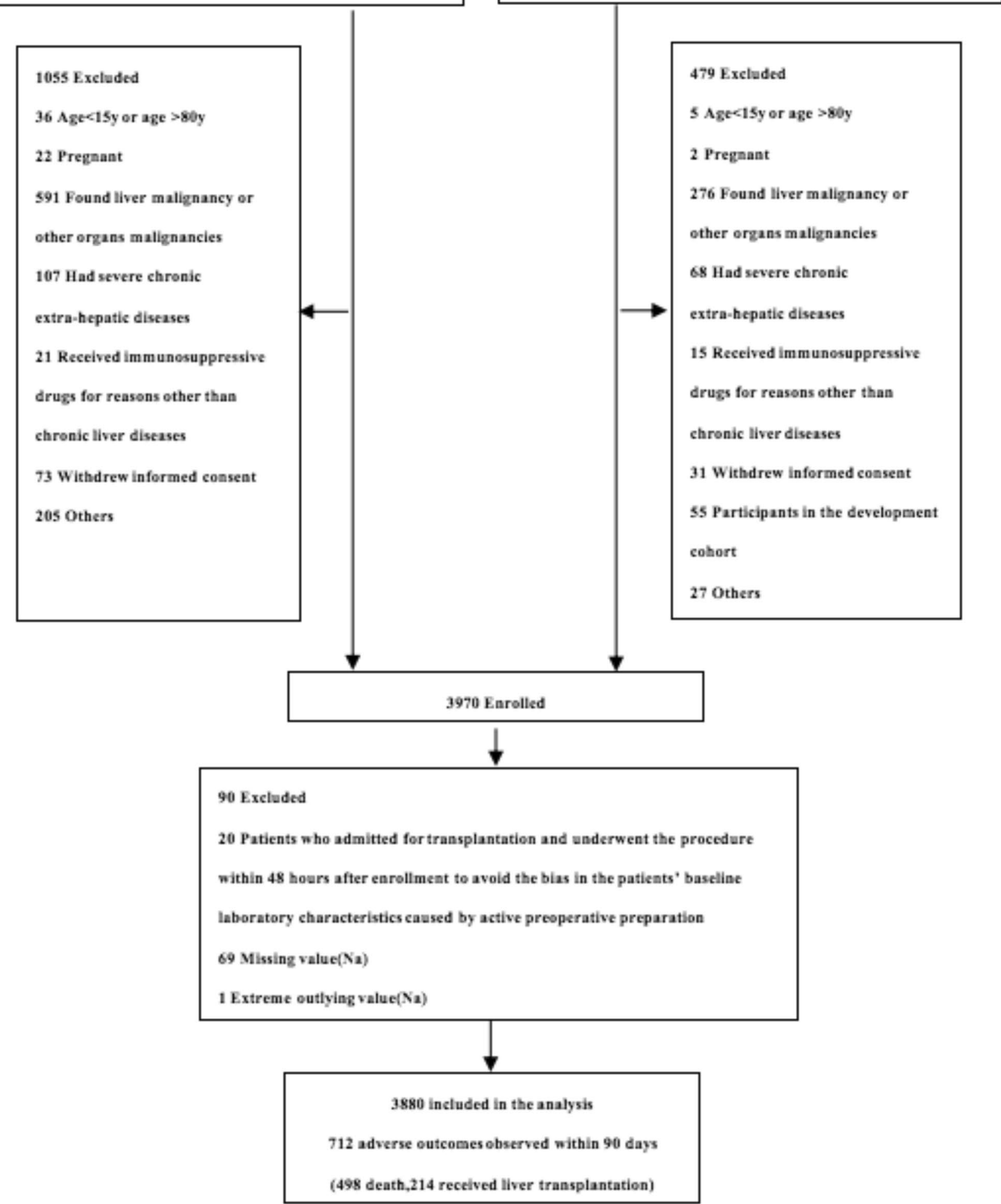

Fig. 1 Flow chart of all enrolled patients after applying the inclusion and exclusion criteria 
Table 1 Baseline characteristics of patients with AoCLD among different Na level groups

\begin{tabular}{|c|c|c|c|c|c|}
\hline Characteristics & $\begin{array}{l}\mathrm{Na} \leq 120 \mathrm{mmol} / \mathrm{L} \\
(n=28)\end{array}$ & $\begin{array}{l}120<\mathrm{Na}<135 \mathrm{mmol} / \mathrm{L} \\
(n=813)\end{array}$ & $\begin{array}{l}135 \leq \mathrm{Na} \leq 145 \mathrm{mmol} / \mathrm{L} \\
(n=2959)\end{array}$ & $\begin{array}{l}\mathrm{Na}>145 \mathrm{mmol} / \mathrm{L} \\
(n=80)\end{array}$ & $p$ value \\
\hline \multicolumn{6}{|l|}{ Demographics } \\
\hline Age (years) & $51.57[43.14-59.28]$ & $50.86[43-58.85]$ & $47.96[39.15-57.13]$ & 55 [43.38-62] & 0.000 \\
\hline Sex (male) & $16(57.1)$ & $621(76.4)$ & $2170(73.3)$ & $51(63.7)$ & 0.010 \\
\hline Etiology & & & & & 0.160 \\
\hline Alcoholic & $7(25)$ & $95(11.7)$ & $229(7.7)$ & $8(10)$ & 0.000 \\
\hline HBV & $11(39.3)$ & $429(52.8)$ & $1705(57.6)$ & $41(51.2)$ & 0.016 \\
\hline AIH & $2(7.1)$ & $62(7.6)$ & $275(9.3)$ & $11(13.8)$ & 0.228 \\
\hline $\mathrm{HCV}$ & $1(3.6)$ & $20(2.5)$ & $82(2.8)$ & $4(5)$ & 0.669 \\
\hline NAFLD & $0(0)$ & $1(0.1)$ & $38(1.3)$ & $0(0)$ & 0.021 \\
\hline Schistosomiasis & $0(0)$ & $7(0.9)$ & $16(0.5)$ & $2(2.5)$ & 0.134 \\
\hline $\mathrm{HBV}+$ Alcoholic & $4(14.3)$ & $102(12.5)$ & $241(8.1)$ & $4(5)$ & 0.001 \\
\hline $\mathrm{HBV}+\mathrm{AIH}$ & $0(0)$ & $4(0.5)$ & $9(0.3)$ & $0(0)$ & 0.790 \\
\hline $\mathrm{HBV}+\mathrm{HCV}$ & $0(0)$ & $5(0.6)$ & $10(0.3)$ & $1(1.3)$ & 0.441 \\
\hline HBV + NAFLD & $0(0)$ & $12(1.5)$ & 87 (2.9) & $1(1.3)$ & 0.079 \\
\hline $\mathrm{HBV}+\mathrm{HEV}$ & $1(3.6)$ & $11(1.4)$ & $58(2)$ & $3(3.8)$ & 0.351 \\
\hline $\mathrm{HBV}+$ Schistosomiasis & $1(3.6)$ & $5(0.6)$ & $17(0.6)$ & $0(0)$ & 0.194 \\
\hline \multicolumn{6}{|l|}{ Cirrhosis status } \\
\hline non-cirrhosis & $1(3.6)$ & $114(14)$ & 969 (32.7) & $22(27.5)$ & 0.000 \\
\hline compensated cirrhosis & $8(28.6)$ & $370(45.5)$ & $1221(41.3)$ & $26(32.5)$ & 0.000 \\
\hline decompensated cirrhosis & $19(67.9)$ & $329(40.5)$ & $769(26)$ & $32(40)$ & 0.000 \\
\hline \multicolumn{6}{|l|}{ Acute decompensation } \\
\hline $\mathrm{HE}$ & & & & & 0.000 \\
\hline Grade 0 & $22(78.6)$ & $709(87.2)$ & $2758(93.2)$ & $56(30)$ & \\
\hline Grade 1 & $2(7.1)$ & $27(3.3)$ & $92(3.1)$ & $5(6.3)$ & \\
\hline Grade 2 & $4(14.3)$ & $50(6.2)$ & $72(2.4)$ & $10(12.5)$ & \\
\hline Grade 3 & $0(0)$ & $23(2.8)$ & $26(0.9)$ & $5(6.3)$ & \\
\hline Grade 4 & $0(0)$ & $4(0.5)$ & $11(0.4)$ & $4(5)$ & \\
\hline Infection & $10(35.7)$ & $306(37.6)$ & $511(17.3)$ & $12(15)$ & 0.000 \\
\hline Ascites & $17(60.7)$ & $546(67.2)$ & $1236(41.8)$ & $32(40)$ & 0.000 \\
\hline Gastrointestinal bleeding & $4(14.3)$ & $113(13.9)$ & $436(14.7)$ & $15(18.8)$ & 0.707 \\
\hline Jaundice & $20(71.4)$ & $543(66.8)$ & $1250(42.2)$ & $22(27.5)$ & 0.000 \\
\hline \multicolumn{6}{|l|}{ Laboratory tests } \\
\hline $\operatorname{ALT}(\mathrm{U} / \mathrm{L})$ & 73.3 [39.23-135.63] & $60.2[29.8-192]$ & $106[33.1-472]$ & $53[26-241]$ & 0.000 \\
\hline AST (U/L) & 113.75 [41.28-172.9] & 96 [49.5-208.05] & 112 [47-299.35] & $84.9[35.6-161]$ & 0.000 \\
\hline ALB (g/L) & $30.4[26.4-35.08]$ & 29.6 [25.7-33.25] & $32.9[28.8-37.4]$ & $32.9[28.25-37.6]$ & 0.000 \\
\hline TBIL (mg/dL) & $12.21[3.37-26.84]$ & 11.11 [3.19-22.96] & $3.42[1.44-12.14]$ & 2.04 [1.25-6.57] & 0.000 \\
\hline INR & 2.04 [1.37-2.69] & 1.68 [1.36-2.23] & $1.37[1.16-1.7]$ & $1.31[1.16-1.62]$ & 0.000 \\
\hline $\mathrm{CR}(\mathrm{mg} / \mathrm{dL})$ & $0.84[0.58-1.35]$ & $0.81[0.67-1.08]$ & $0.76[0.63-0.91]$ & $0.8[0.6-0.96]$ & 0.000 \\
\hline BUN (mmol/L) & 9.53 [4.57-14.95] & $5.81[4.04-8.6]$ & $4.3[3.3-5.89]$ & $5.3[3.83-9.23]$ & 0.000 \\
\hline $\mathrm{WBC}(\times 10 * 9 / \mathrm{L})$ & 7.38 [3.6-11.67] & $6.2[4.17-9.21]$ & $4.8[3.5-6.54]$ & $4.38[3.15-5.82]$ & 0.000 \\
\hline PLT $(\times 10 * 9 / \mathrm{L})$ & $67[45.5-122]$ & $79[52-121]$ & $98[60-150.2]$ & $74[51-131]$ & 0.000 \\
\hline $\mathrm{HB}(\mathrm{g} / \mathrm{L})$ & 106.5 [81.5-128.3] & 108 [86-127] & 120 [99-137] & 117 [92-130] & 0.000 \\
\hline $\mathrm{K}(\mathrm{mmol} / \mathrm{L})$ & $4.47[3.52-5.05]$ & $3.9[3.5-4.3]$ & 3.85 [3.54-4.17] & $3.8[3.3-4.1]$ & 0.093 \\
\hline $\mathrm{PaO}_{2} / \mathrm{FiO}_{2}$ & 476.19 [466.67-476.19] & 471.43 [466.67-476.19] & 476.19 [466.67-476.19] & 476.19 [466.67-476.19] & 0.000 \\
\hline MAP (mmHg) & 86.67 [80.5-93.33] & 88 [80.67-93.33] & $89[83.33-95]$ & $93.33[86.67-98]$ & 0.000 \\
\hline \multicolumn{6}{|l|}{ Scores } \\
\hline MELD & $23[17.5-31]$ & $21[15-27]$ & $15[10-21]$ & 12 [10-17] & 0.000 \\
\hline MELD_Na & 33 [30.25-37] & 25 [20-30] & 15 [10-22] & $7[5-13]$ & 0.000 \\
\hline iMELD & 55 [51-64.5] & $44[38-50]$ & 32 [26-38] & 26 [23-33] & 0.000 \\
\hline
\end{tabular}


Table 1 (continued)

\begin{tabular}{|c|c|c|c|c|c|}
\hline Characteristics & $\begin{array}{l}\mathrm{Na} \leq 120 \mathrm{mmol} / \mathrm{L} \\
(n=28)\end{array}$ & $\begin{array}{l}120<\mathrm{Na}<135 \mathrm{mmol} / \mathrm{L} \\
(n=813)\end{array}$ & $\begin{array}{l}135 \leq \mathrm{Na} \leq 145 \mathrm{mmol} / \mathrm{L} \\
(n=2959)\end{array}$ & $\begin{array}{l}\mathrm{Na}>145 \mathrm{mmol} / \mathrm{L} \\
(n=80)\end{array}$ & $p$ value \\
\hline CLIF_SOFA & $6[5.25-8]$ & $7[5-7]$ & $4[2-6]$ & $4[2-7]$ & 0.000 \\
\hline SOFA & $10[9-11]$ & $9[8-10]$ & $8[6-9]$ & 7 [7-9] & 0.000 \\
\hline CHILD_PUGH & $10.5[9-11.75]$ & $10[9-11]$ & $8[7-10]$ & $8[7-10]$ & 0.000 \\
\hline \multicolumn{6}{|l|}{ Outcomes } \\
\hline 90-day adverse outcomes & $14(50)$ & $286(35.2)$ & $398(13.5)$ & $14(17.5)$ & 0.000 \\
\hline 90-day death & $13(46.4)$ & $212(26.1)$ & $261(8.8)$ & $12(15)$ & 0.000 \\
\hline 90-day LT & $1(3.6)$ & 74 (9.1) & 137 (4.6) & $2(2.5)$ & 0.000 \\
\hline
\end{tabular}

$N a$ serum sodium, $H B V$ hepatitis B virus, $A I H$ autoimmune hepatitis, $H C V$ hepatitis $\mathrm{C}$ virus, $H E$ hepatic encephalopathy, $A L T$ aspartate aminotransferase, $A S T$ alanine aminotransferase, $A L B$ albumin, $T B I L$ total bilirubin, INR international normalized ratio, $C R$ serum creatinine, $B U N$ blood urea nitrogen, $W B C$ white blood cell, $H B$ hemoglobin, $P L T$ platelet, $\mathrm{K}$ serum potassium, $\mathrm{PaO} 2$ arterial partial pressure of oxygen, $\mathrm{FiO} 2$ fraction of inspired O2, MAP mean arterial pressure, MELD model for end-stage liver disease, iMELD integrated MELD, SOFA Sequential Organ Failure Assessment, CLIF-SOFA Chronic Liver Failure Assessment, Child-Pugh Child-Turcotte-Pugh, LT liver transplantation

*Continuous variables are presented as median (interquartile range); categorical variables are presented as $\mathrm{n}$ (percentage)

Table 2 Adjusted effects of Na level on 90-day adverse outcomes (death or LT) in all patients $(n=3880)$

\begin{tabular}{|c|c|c|c|c|c|}
\hline & $\begin{array}{l}\text { Number of 90-day } \\
\text { adverse outcomes } \\
\text { (percentage) }\end{array}$ & $\begin{array}{l}\text { OR, 95\% CI, } p \text { value } \\
\text { Model I }\end{array}$ & $\begin{array}{l}\text { OR, } 95 \% \text { CI, } p \text { value } \\
\text { Model II }\end{array}$ & $\begin{array}{l}\text { OR, 95\% CI, } p \text { value } \\
\text { Model III }\end{array}$ & $\begin{array}{l}\text { OR, } 95 \% \text { CI, } p \text { value } \\
\text { Model IV }\end{array}$ \\
\hline $\mathrm{Na}$ (continuous) & $712(18.35)$ & $\begin{array}{l}0.88 \\
\quad(0.87,0.90)<0.001\end{array}$ & $\begin{array}{l}0.90 \\
\quad(0.88,0.91)<0.001\end{array}$ & $\begin{array}{l}0.91 \\
\quad(0.89,0.92)<0.001\end{array}$ & $0.95(0.93,0.97)<0.001$ \\
\hline \multicolumn{6}{|l|}{ Na-categorical } \\
\hline$(\mathrm{Na} 135-145 \mathrm{mmol} / \mathrm{L})$ & $398(13.5)$ & 1 & 1 & 1 & 1 \\
\hline$(\mathrm{Na}>145 \mathrm{mmol} / \mathrm{L})$ & $14(17.5)$ & $1.37(1.02,1.83) 0.039$ & $1.30(0.72,2.36) 0.386$ & $0.91(0.89,1.85) 0.991$ & $1.21(0.63,2.34) 0.565$ \\
\hline (Na $120-135$ mmol/L) & $286(35.2)$ & $\begin{array}{l}3.49 \\
\quad(2.92,4.18)<0.001\end{array}$ & $\begin{array}{l}3.02 \\
\quad(2.52,3.63)<0.001\end{array}$ & $\begin{array}{l}2.56 \\
\quad(2.12,3.09)<0.001\end{array}$ & $1.72(1.39,2.13)<0.001$ \\
\hline$(\mathrm{Na} \leq 120 \mathrm{mmol} / \mathrm{L})$ & $14(50)$ & $\begin{array}{l}6.44 \\
\quad(3.05,13.60)<0.001\end{array}$ & $\begin{array}{l}5.19 \\
\quad(2.45,11.03)<0.001\end{array}$ & $\begin{array}{l}4.41 \\
\quad(2.03,9.58)<0.001\end{array}$ & $2.36(0.98,5.67) 0.056$ \\
\hline$p$ value for trend* & & $<0.001$ & $<0.001$ & $<0.001$ & $<0.001$ \\
\hline $\begin{array}{l}\mathrm{Na} \text { (continuous } \\
5 \mathrm{mmol} / \mathrm{L} \text { decrease) }\end{array}$ & & $\begin{array}{l}0.54 \\
\quad(0.50,0.59)<0.001\end{array}$ & $\begin{array}{l}0.58 \\
\quad(0.53,0.63)<0.001\end{array}$ & $\begin{array}{l}0.62 \\
\quad(0.57,0.67)<0.001\end{array}$ & $0.76(0.70,0.84)<0.001$ \\
\hline $\begin{array}{l}\mathrm{Na} \text { (continu- } \\
\text { ous } 10 \mathrm{mmol} / \mathrm{L} \\
\text { decrease) }\end{array}$ & & $\begin{array}{l}0.29 \\
\quad(0.25,0.34)<0.001\end{array}$ & $\begin{array}{l}0.33 \\
\quad(0.28,0.39)<0.001\end{array}$ & $\begin{array}{l}0.38 \\
\quad(0.32,0.45)<0.001\end{array}$ & $0.58(0.49,0.70)<0.001$ \\
\hline
\end{tabular}

Model I Un-adjusted;

Model II Adjusted for age, sex, cirrhosis;

Model III Adjusted for age, sex, cirrhosis, HE, ascites, infection;

Model IV Adjusted for age, sex, cirrhosis, HE, ascites, infection, TBIL, INR, CR;

CI confidence interval

*Test for trend based on variables containing median value for each group

95\% CI 2.50-3.62; $p<0.001$ ) (Fig. 2c), regardless of cirrhosis (Supplementary Fig. 4a, b).

\section{Dynamic changes in $\mathrm{Na}$ level on days 4 and 7 related to 90-day adverse outcomes}

If the $\mathrm{Na}$ level of patients increased to $>135 \mathrm{mmol} / \mathrm{L}$, hyponatremia was corrected. The 90 -day survival rate was significantly higher in the corrected group than in the uncorrected group, regardless of the correction of hyponatremia on days 4 or 7 after admission ( $75.2 \%$ vs. $56.1 \%$ and $75.4 \%$ vs. $66.4 \% ; p<0.05$ ) (Fig. 2d, e).

On day 4, the MELD, SOFA, and Child-Pugh scores, baseline WBC, and baseline TBIL, INR, and CR levels in the uncorrected group were significantly higher than those in the corrected group. The corrected group had a lower 
Table 3 Adjusted effects of Na level on 90-day adverse outcomes (death or LT) in cirrhosis patients ( $n=2774$ )

\begin{tabular}{|c|c|c|c|c|c|}
\hline & $\begin{array}{l}\text { Number of 90-day } \\
\text { adverse outcomes } \\
\text { (percentage) }\end{array}$ & $\begin{array}{l}\text { OR, 95\% CI, } p \text { value } \\
\text { Model I }\end{array}$ & $\begin{array}{l}\text { OR, } 95 \% \text { CI, } p \text { value } \\
\text { Model II }\end{array}$ & $\begin{array}{l}\text { OR, 95\% CI, } p \text { value } \\
\text { Model III }\end{array}$ & $\begin{array}{l}\text { OR, } 95 \% \text { CI, } p \text { value } \\
\text { Model IV }\end{array}$ \\
\hline $\mathrm{Na}$ (continuous) & $626(22.57)$ & $\begin{array}{l}0.90 \\
\quad(0.88,0.91)<0.001\end{array}$ & $\begin{array}{l}0.90 \\
\quad(0.88,0.91)<0.001\end{array}$ & $\begin{array}{l}0.91 \\
\quad(0.89,0.92)<0.001\end{array}$ & $0.95(0.93,0.96)<0.001$ \\
\hline \multicolumn{6}{|l|}{ Na-categorical } \\
\hline (Na 135-145 mmol/L) & $336(16.9)$ & 1 & 1 & 1 & 1 \\
\hline$(\mathrm{Na}>145 \mathrm{mmol} / \mathrm{L})$ & $11(19)$ & $1.15(0.59,2.24) 0.677$ & $1.17(0.60,2.29) 0.641$ & $0.92(0.47,1.83) 0.822$ & $1.20(0.58,2.50) 0.623$ \\
\hline (Na 120-135 mmol/L) & $266(38.1)$ & $\begin{array}{l}3.02 \\
\quad(2.50,3.67)<0.001\end{array}$ & $\begin{array}{l}3.02 \\
\quad(2.49,3.66)<0.001\end{array}$ & $\begin{array}{l}2.64 \\
\quad(2.16,3.23)<0.001\end{array}$ & $1.78(1.42,2.23)<0.001$ \\
\hline$(\mathrm{Na} \leq 120 \mathrm{mmol} / \mathrm{L})$ & $13(48.1)$ & $\begin{array}{l}4.57 \\
\quad(2.13,9.81)<0.001\end{array}$ & $\begin{array}{l}4.64 \\
\quad(2.16,9.96)<0.001\end{array}$ & $\begin{array}{l}4.01 \\
\quad(1.84,8.74)<0.001\end{array}$ & $2.13(0.86,5.24) 0.100$ \\
\hline$p$ value for trend $*$ & & $<0.001$ & $<0.001$ & $<0.001$ & $<0.001$ \\
\hline $\begin{array}{l}\mathrm{Na} \text { (continuous } \\
5 \mathrm{mmol} / \mathrm{L} \text { decrease) }\end{array}$ & & $\begin{array}{l}0.58 \\
\quad(0.53,0.63)<0.001\end{array}$ & $\begin{array}{l}0.58 \\
\quad(0.53,0.63)<0.001\end{array}$ & $\begin{array}{l}0.6 \\
\quad(0.56,0.67)<0.001\end{array}$ & $0.75(0.68,0.83)<0.001$ \\
\hline $\begin{array}{l}\mathrm{Na} \text { (continu- } \\
\text { ous } 10 \mathrm{mmol} / \mathrm{L} \\
\text { decrease) }\end{array}$ & & $\begin{array}{l}0.34 \\
\quad(0.28,0.40)<0.001\end{array}$ & $\begin{array}{l}0.34 \\
\quad(0.28,0.40)<0.001\end{array}$ & $\begin{array}{l}0.3 \\
\quad(0.31,0.45)<0.001\end{array}$ & $0.57(0.47,0.69)<0.001$ \\
\hline
\end{tabular}

Model I Un-adjusted;

Model II Adjusted for age, sex;

Model III Adjusted for age, sex, HE, ascites, infection;

Model IV Adjusted for age, sex, HE, ascites, infection, TBIL, INR, CR;

CI confidence interval

*Test for trend based on variables containing median value for each group

Table 4 Adjusted effects of Na level on 90-day adverse outcomes (death or LT) in the non-cirrhotic patients $(n=1106)$

\begin{tabular}{|c|c|c|c|c|c|}
\hline & $\begin{array}{l}\text { Number of 90-day } \\
\text { adverse outcomes } \\
\text { (percentage) }\end{array}$ & $\begin{array}{l}\text { OR,95\% CI, } p \text { value } \\
\text { Model I }\end{array}$ & $\begin{array}{l}\text { OR, } 95 \% \text { CI, } p \text { value } \\
\text { Model II }\end{array}$ & $\begin{array}{l}\text { OR, } 95 \% \text { CI, } p \text { value } \\
\text { Model III }\end{array}$ & $\begin{array}{l}\text { OR, } 95 \% \text { CI, } p \text { value } \\
\text { Model IV }\end{array}$ \\
\hline $\mathrm{Na}$ (continuous) & $86(7.78)$ & $\begin{array}{l}0.88 \\
\quad(0.84,0.94)<0.001\end{array}$ & $\begin{array}{l}0.89 \\
\quad(0.84,0.94)<0.001\end{array}$ & $0.93(0.88,0.99) 0.023$ & $0.99(0.93,1.05) 0.659$ \\
\hline \multicolumn{6}{|l|}{$\mathrm{Na}($ categorical $)$} \\
\hline $\begin{array}{l}\text { (Na level 135- } \\
145 \mathrm{mmol} / \mathrm{L})\end{array}$ & $62(6.4)$ & 1 & 1 & 1 & 1 \\
\hline $\begin{array}{l}(\mathrm{Na} \\
\quad \text { level > } 145 \mathrm{mmol} / \mathrm{L})\end{array}$ & $3(13.6)$ & $2.31(0.67,8.02) 0.187$ & $2.22(0.63,7.84) 0.215$ & $1.98(0.44,8.89) 0.371$ & $1.69(0.36,7.98) 0.505$ \\
\hline $\begin{array}{l}\text { (Na level 120- } \\
135 \mathrm{mmol} / \mathrm{L})\end{array}$ & $21(18.2)$ & $\begin{array}{l}3.27 \\
\quad(1.91,5.60)<0.001\end{array}$ & $2.9(1.69,5.04)<0.001$ & $2.06(1.10,3.83) 0.024$ & $1.47(0.76,2.81) 0.250$ \\
\hline$p$ value for trend* & & $<0.001$ & $<0.001$ & 0.023 & 0.207 \\
\hline $\begin{array}{l}\mathrm{Na} \text { (Continuous } \\
5 \mathrm{mmol} / \mathrm{L} \text { decrease })\end{array}$ & & $\begin{array}{l}0.54 \\
\quad(0.41,0.72)<0.001\end{array}$ & $\begin{array}{l}0.56 \\
\quad(0.43,0.74)<0.001\end{array}$ & $0.71(0.53,0.96) 0.023$ & $0.93(0.68,1.28) 0.659$ \\
\hline $\begin{array}{l}\mathrm{Na} \text { (Continuous } \\
10 \mathrm{mmol} / \mathrm{L} \text { decrease) }\end{array}$ & & $\begin{array}{l}0.29 \\
\quad(0.17,0.51)<0.001\end{array}$ & $\begin{array}{l}0.32 \\
\quad(0.18,0.55)<0.001\end{array}$ & $0.51(0.28,0.91) 0.023$ & $0.87(0.46,1.63) 0.659$ \\
\hline
\end{tabular}

Model I Un-adjusted;

Model II Adjusted for age, sex;

Model III Adjusted for age, sex, HE, ascites, infection;

Model IV Adjusted for age, sex, HE, ascites, infection, TBIL, INR, CR;

CI confidence interval

* Test for trend based on variables containing median value for each group 
a

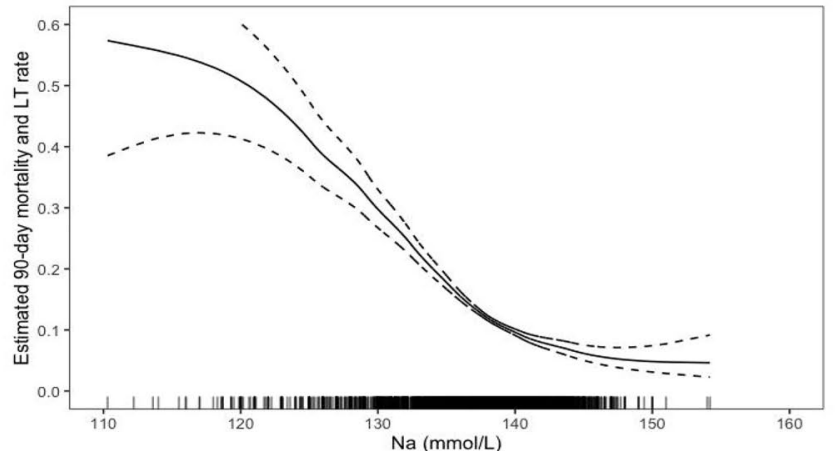

C

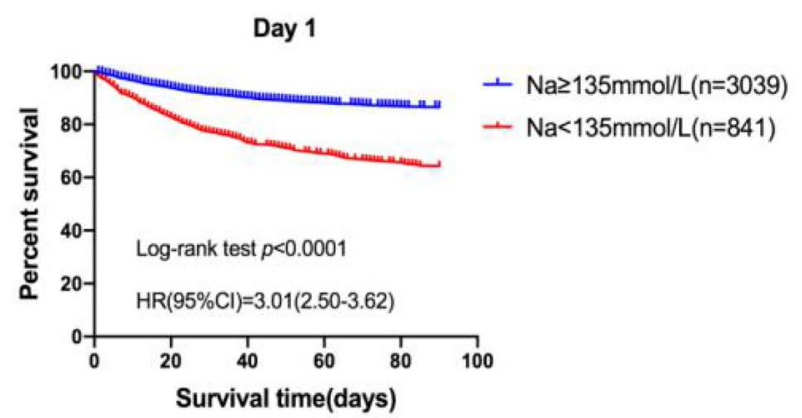

b

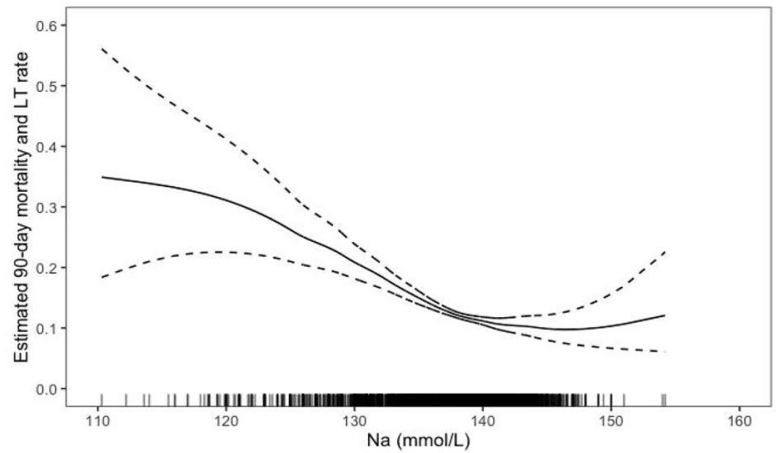

d

Day 4

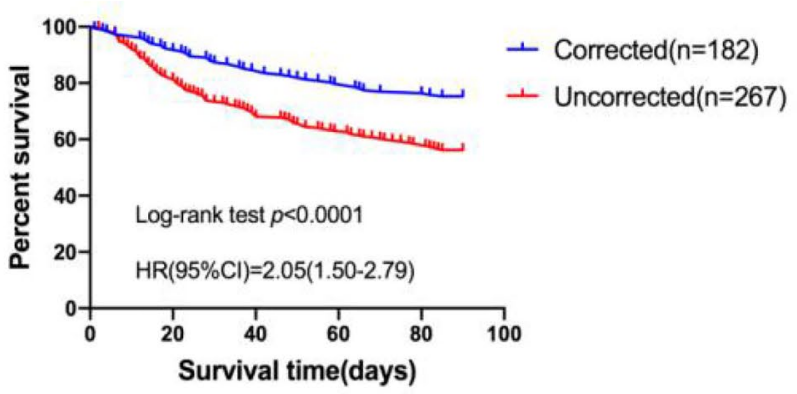

e

\begin{tabular}{|c|c|}
\hline \multicolumn{2}{|c|}{ After adjusted } \\
\hline Number & Percentage survival \\
\hline 541 & $64.3 \%$ \\
\hline 2627 & $86.4 \%$ \\
\hline 3168 & $81.6 \%$ \\
\hline
\end{tabular}

\begin{tabular}{|c|c|c|c|c|}
\hline \multirow{2}{*}{ bodium_cutoff } & \multirow{2}{*}{ Total number } & \multirow{2}{*}{ Adverse outcome } & \multicolumn{2}{|c|}{ After adjusted } \\
\hline & & & Number & Percentage survival \\
\hline$<135$ & 267 & 117 & 150 & $56.1 \%$ \\
\hline$>=135$ & 182 & 45 & 137 & $75.2 \%$ \\
\hline All & 449 & 162 & 287 & $63.9 \%$ \\
\hline
\end{tabular}

f

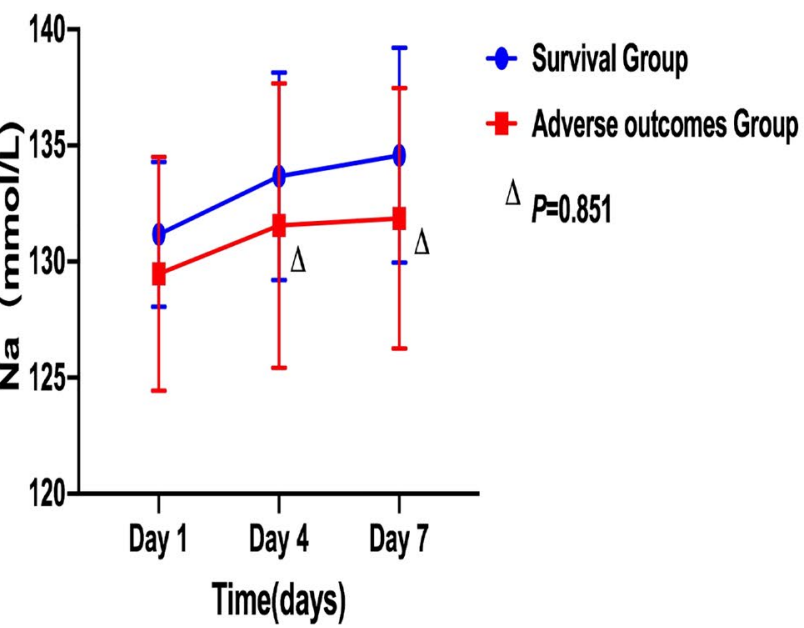


4Fig. 2 a The association between Na level and 90-day adverse outcomes in patients with AoCLD (unadjusted). b The association between $\mathrm{Na}$ level and 90-day adverse outcomes in patients with AoCLD (adjusted). c Survival analysis, based on the Na cut-off value, using the Kaplan-Meier method. d Kaplan-Meier curves of patients with corrected or uncorrected hyponatremia on day 4 after admission. e Kaplan-Meier curves of patients with corrected or uncorrected hyponatremia on day 7 after admission. f Dynamic changes in $\mathrm{Na}$ level between the survival group and adverse outcome group 7 days after admission

incidence of cirrhosis, ascites, and infection than the uncorrected group $(p<0.05)$ (Supplementary Table 4$)$. Dynamic observation revealed that the Na levels on days 4 and 7 were significantly lower in patients with 90-day adverse outcomes after admission than in patients in the survival group $(p<0.05)$. However, in the 90-day adverse outcomes group, changes in $\mathrm{Na}$ level were not significantly different between days 4 and 7 ( $p=0.851$ ) (Fig. 2f).

\section{Discussion}

Our study population represents most inpatients with liver disease in areas with a high prevalence of HBV in China. Therefore, despite the fact that hyponatremia is a well-recognized complication of cirrhotic patients, a specific analysis of hyponatremia in patients with AoCLD had so far not been reported. The investigation of this relationship is clinically relevant, given the important physiological effects of low serum Na levels and the well-demonstrated relationship between hyponatremia and survival in a Chinese population with CLD. To the best of our knowledge, this study is the first to describe the prevalence of hyponatremia, normonatremia, and hypernatremia and their relationship with 90-day prognosis in hospitalized patients with AoCLD. We confirmed that hyponatremia is an independent risk factor for 90-day adverse outcomes in this population, which is independent of other indicators reflecting liver dysfunction, regardless of the etiology of chronic liver disease and whether patients with hepatitis B have received NUCs before admission. The risk of 90-day adverse outcomes decreased by $5 \%, 24 \%$, and $42 \%$ for Na levels increased by 1,5 , and $10 \mathrm{mmol} / \mathrm{L}$, respectively. These patients whose hyponatremia could be corrected on days 4 or 7 had a better prognosis than those whose hyponatremia could not be corrected.

In the past few decades, major studies [7, 9-12] have confirmed that hyponatremia is an independent risk factor for poor prognosis in cirrhotic patients. The European CANONIC cohort study in 2014 indicated that hyponatremia was also an independent risk factor for 90-day poor prognosis in patients with acute-on-chronic liver failure [22]. As expected, our study showed similar results in hospitalized patients with AoCLD. The LT-free mortality of patients with severe hyponatremia in our study (48\%) was higher than that reported in a previous study [23]. In advanced cirrhosis, renal free water clearance function is impaired, serum $\mathrm{Na}$ balance is not maintained, and diluted hyponatremia occurs and is reasonably considered present; however, due to its unique human physiopathological mechanisms of systemic vasodilatation, clinicians often ignore it in daily practice [12]. Previous research showed that hyponatremia is usually accompanied by various complications in cirrhotic patients, which somewhat reflect the severity of portal hypertension [19, 24]. In our study, the incidence of liver cirrhosis, ascites, infection, hepatic encephalopathy, and jaundice in hyponatremic patients was also significantly higher, especially in patients with severe hyponatremia. Patients with severe hyponatremia tended to have a poorer liver function and higher 90-day mortality. Thus, LT should be actively considered for this population. Moreover, it is worth mentioning that $18.2 \%$ of noncirrhotic patients in our cohort still had hyponatremia and a risk of 90-day adverse outcomes. This number was only slightly lower than that in cirrhotic patients. Interestingly, after adjusting for acute decompensated events in noncirrhotic patients, the risk of 90-day adverse outcomes was statistically significant. However, the risk disappeared after the MELD score was adjusted, which suggested that Na level was not a predictor of 90-day prognosis in noncirrhotic patients. This finding was different from that in cirrhotic patients as the mechanisms of hyponatremia differ.

The European Association for the Study of the Liver guidelines (Clinical Practice Guidelines for the Management of Patients with Decompensated Cirrhosis) recommends that cirrhotic patients limit fluid intake and increase the dose of diuretics or use vaptans to increase the excretion of renal solute free water and other measures to improve hyponatremia when $\mathrm{Na}$ level is $<130 \mathrm{mmol} / \mathrm{L}$ [25]. In fact, high-level hyponatremia ( $\mathrm{Na}$ level, $130-135 \mathrm{mmol} / \mathrm{L}$ ) is deemed unsuitable for treatment because it is always considered a frequent and harmless feature of severe liver disease [26]. In our study, there were 86 patients with $\mathrm{Na}$ level of $125 \mathrm{mmol} / \mathrm{L}$ and 755 patients with $\mathrm{Na}$ level between $>125 \mathrm{mmol} / \mathrm{L}$ and $<135 \mathrm{mmol} / \mathrm{L}$. However, when $\mathrm{Na}$ level was $<135 \mathrm{mmol} / \mathrm{L}$, the risk of 90-day adverse outcomes increased, which was stricter than the $130 \mathrm{mmol} / \mathrm{L}$ recommended by the guidelines [25]. This finding suggests the necessity of hyponatremia correction in patients with AoCLD. Na level of $<135 \mathrm{mmol} / \mathrm{L}$ should be considered for management by clinicians. Ahluwalia et al.[27] found that cirrhotic patients can benefit from hyponatremia correction, which improves cognitive function and HRQOL. Dedicated specialist input and active treatment of severe hyponatremia can effectively reduce mortality [28]. Based on experience from the European CANONIC cohort study, most of the patients will have a clear prognosis between days 3 and 7 
of hospital admission, and clinical decisions, such as evaluation for liver transplant or discussion over goals of care, could be tailored using clinical scores [29]. However, there is limited information regarding whether hyponatremia correction in 1 week could improve the 90-day prognosis. Our study suggests that hyponatremia correction was associated with improvement in clinically relevant outcomes, regardless of day 4 or 7 . The Na level increased continuously from day 4 to 7 in hyponatremic patients who survived; therefore, focusing on dynamic changes in Na level and managing hyponatremia in AoCLD in the first week after admission are crucial. Persistent abnormal $\mathrm{Na}$ level is often associated with an increased risk of death. This finding is supported by the fact that hyponatremia could not be corrected on day 4 , and hyponatremic patients had a higher mortality rate, MELD score, and incidence of cirrhosis, infection, and ascites than those without hyponatremia. Therefore, managing complications associated with hyponatremia is essential. However, this needs to be further studied in patients with AoCLD and those with hyponatremia.

This was a large multicenter, prospective cohort study focused on areas with a high prevalence of HBV in China. All patients were followed up for 90 days, and most data were collected to ensure authenticity and integrity. In addition to the baseline material, we similarly analyzed the dynamic $\mathrm{Na}$ levels on days 4 and 7 after admission.

This study has some limitations. First, there were some missing values for the dynamic Na level. Second, owing to the nature of observational studies and various patient conditions, therapy measures of hyponatremia were not unified among the centers; however, we employed a unified standard of diagnosis and treatment for hyponatremia [30].

In conclusion, our study showed that hyponatremia is an independent risk factor for 90-day adverse outcomes in hospitalized patients with AoCLD. Elevated Na levels reduced the risk of 90-day adverse outcomes, and failure to correct hyponatremia in a week after admission was often associated with increased mortality. Therefore, clinicians should focus on hyponatremia in patients with AoCLD and actively manage its related complications in daily clinical practice for hyponatremia correction.

Supplementary Information The online version contains supplementary material available at https://doi.org/10.1007/s12072-021-10282-8.

Acknowledgements We thank the following Chinese (Acute on) Chronic Liver Failure Consortium (Ch-CLIF.C) members and participants for the contributions to this study: Department of Liver Intensive Care Unit, Shanghai Public Health Clinical Centre, Fudan UniversityJiefei Wang, Wei Yuan; Department of Gastroenterology, Ren Ji Hospital, School of Medicine, Shanghai Jiao Tong University-Shan Yin, Wenyi Gu, Yan Zhang, Tongyu Wang, Dandan Wu, Fuchen Dong, Bo Zeng, Liuying Chen, Shijin Wang; Clinical Research Institute, Shanghai Jiao Tong University School of Medicine, Shanghai, China, Zhang Weituo; Centre of Integrative Medicine, Beijing Ditan Hospital, Capital
Medical University-Qun Zhang, Yixin Hou, Yuxin Li, Yunyi Huang; Department of Infectious Diseases, Southwest Hospital, Third Military Medical University (Army Medical University)—Shuning Sun, Wenting Tan, Xiaomei Xiang, Yunjie Dan; Department of Infectious Disease, Hunan Key Laboratory of Viral Hepatitis, Xiangya Hospital, Central South University-Jun Chen, Ruochan Chen, Liyuan Long; Department of Infectious Diseases, Institute of Infection and Immunology, Union Hospital, Tongji Medical College, Huazhong University of Science and Technology_ Jing Liu, Ling Xu, Shue Xiong, Yan Xiong, Congcong Zou; Hepatology Unit, Department of Infectious Diseases, Nanfang Hospital, Southern Medical University-Renjie Ouyang, Congyan Zhu; Department of Hepatology, First Hospital of Jilin University-Chang Jiang, Xiaoyu Wen, Na Gao, Chunyan Liu; Department of Infectious Disease, Taihe Hospital, Hubei University of Medicine-Ying Wang, Qing Lei, Sen Luo; Department of Infectious Disease, The First Hospital of Zhejiang University-Haotang Ren; Department of Infectious Diseases and Hepatology, Second Hospital of Shandong University_-Tao Li, Xuanqiong Fang, Jing Li, Ziyu Wang; Liver Disease Centre, First Affiliated Hospital of Xinjiang Medical University-Rongjiong Zheng, Fangrong Jie, Nan Li; Department of Infectious Disease, Henan Provincial People's Hospital-Huiming Jin; Infectious Disease Center, Affiliated Hospital of Logistics University of People's Armed Police Force-Hai Li, Qing Zhang, Xuequn Zheng; and Department of Infectious Disease, Fuzhou General Hospital of Nanjing Military Command-Shaoyang Wang, Taofa Lin.

Author contributions XM, HL, XW, GD, and YH contributed equally and share first authorship. HL, GD, XW, XZ, YH, JC, ZM, YG, FL, $\mathrm{XL}$, YS, YZ, HY, and ZQ obtained the funding. ZQ and WY designed the study. LQ, WG, YZ, XX, YZ, SS, YH, QZ, YX, CZ, JC, ZH, BL, XJ, GZ, HW, YC, SL, NG, CL, JL, TL, RZ, XZ, and HR collected the data. WZ directed statistical analysis. XM drafted the manuscript. ZQ and WY contributed to the critical revision of the manuscript for important intellectual content and approved the final version of the manuscript. All authors have read and approved the final manuscript.

Funding This research was supported by the Shanghai Municipal Education Commission Gaofeng Clinical Medicine Grant Support and Shanghai Hospital Development Center Funding (SHDC2020CR1037B); the National Science and Technology Major Project (2018ZX10302206, 2018ZX10723203, 2018ZX10725506); the Science Foundation of Shanghai Municipal Health Commission (20184Y0058); and the National Key Research and Development Program of China (2017YFC0908104).

Data and material availability All data included in this study are available upon request by contact with the corresponding author.

Code availability All codes used in this study followed the manuals of each $\mathrm{R}$ package, which are fully open accessed.

\section{Declarations}

Conflict of interest Xue Mei, Hai Li, Guohong Deng, Xianbo Wang, Xin Zheng, Yan Huang, Jinjun Chen, Zhongji Meng, Yanhang Gao, Feng Liu, Xiaobo Lu, Yu Shi, Yubao Zheng, Huadong Yan, Weituo Zhang, Liang Qiao, Wenyi Gu, Yan Zhang, Xiaomei Xiang, Yi Zhou, Shuning Sun, Yixin Hou, Qun Zhang, Yan Xiong, Congcong Zou, Jun Chen, Zebing Huang, Beiling Li, Xiuhua Jiang, Guotao Zhong, Haiyu Wang, Yuanyuan Chen, Sen Luo, Na Gao, Chunyan Liu, Jing Li, Tao Li, Rongjiong Zheng, Xinyi Zhou, Haotang Ren, Wei Yuan, Zhiping Qian declare that they have no conflict of interest. 
Ethics approval The ethics committee of Ren Ji Hospital (the lead center of the CATCH-LIFE study), School of Medicine, Shanghai Jiaotong University (Shanghai, China) approved the study [ethics code: (2014)148k and (2016)142k].

Consent to participate Written informed consent was obtained from all patients.

Consent for publication All authors of this manuscript have read and approved the final submitted version and are aware that they are listed as an author on this paper.

\section{References}

1. Wang S, Toy M, Hang Pham TT, et al. Causes and trends in liver disease and hepatocellular carcinoma among men and women who received liver transplants in the US, 2010-2019. PLoS One. 2020;15:e0239393

2. Seto WK, Mandell MS. Chronic liver disease: global perspectives and future challenges to delivering quality health care. PLoS One. 2021;16:0243607

3. GBD. Cirrhosis Collaborators (2020) The global, regional, and national burden of cirrhosis by cause in 195 countries and territories, 1990-2017: a systematic analysis for the Global Burden of Disease Study 2017. Lancet Gastroenterol Hepatol. 2017;5(3):245-266

4. Lippi G, de Oliveira MHS, Henry BM. Chronic liver disease is not associated with severity or mortality in Coronavirus disease 2019 (COVID-19): a pooled analysis. Eur J Gastroenterol Hepatol. 2021;33:114-115

5. Kamath PS, Wiesner RH, Malinchoc M, et al. A model to predict survival in patients with end-stage liver disease. Hepatology. 2001;33:2

6. Russell W, Erick E, Richard F, et al. Model for end-stage liver disease (MELD) and allocation of donor livers. Gastroenterology. 2003;124:91-96

7. Kim WR, Biggins SW, Kremers WK, et al. Hyponatremia and mortality among patients on the liver-transplant waiting list. N Engl J Med. 2008;359:1018-1026

8. Ruf AE, Kremers WK, Chavez LL, et al. Addition of serum sodium into the MELD score predicts waiting list mortality better than MELD alone. Liver Transpl. 2005;11:336-343

9. Biggins SW, Kim WR, Terrault NA, et al. Evidence-based incorporation of serum sodium concentration into MELD. Gastroenterology. 2006;130:1652-1660

10. Lenci I, Milana M, Grassi G, et al. Natremia and liver transplantation: the right amount of salt for a good recipe. World J Hepatol. 2020;12:919-930

11. Neuberger J, Gimson A, Davies M, Liver Advisory Group, et al. UK blood and transplant. Selection of patients for liver transplantation and allocation of donated livers in the UK. Gut. 2008; 57:252-257

12. Alukal JJ, John S, Thuluvath PJ. Hyponatremia in cirrhosis: an update. Am J Gastroenterol. 2020;115(11):1775-1785

13. Ahluwalia V, Wade James B, Leroy T, et al. Differential impact of hyponatremia and hepatic encephalopathy on health-related quality of life and brain metabolite abnormalities in cirrhosis. J Hepatol. 2013;59:467-473
14. Hennrikus E, Ou G, Kinney B, et al. Prevalence, timing, causes, and outcomes of hyponatremia in hospitalized orthopaedic surgery patients. J Bone Jt Surg Am. 2015;97:1824-1832

15. Mannheimer B, Skov J, Falhammar H, et al. Sex-specific risks of death in patients hospitalized for hyponatremia: a populationbased study. Endocrine. 2019;66:660-665

16. Qiao L, Wang X, Deng G, et al. Cohort profile: a multicentre prospective validation cohort of the Chinese Acute-on-Chronic Liver Failure (CATCH-LIFE) study. BMJ Open. 2021;11:e037793

17. Zhang Y, Xu BY, Wang XB, et al. Prevalence and clinical significance of portal vein thrombosis in patients with cirrhosis and acute decompensation. Clin Gastroenterol Hepatol. 2020;18:2564-72.e1

18. Gu WY, Xu BY, Zheng X, et al. Acute-on-chronic liver failure in China: rationale for developing a patient registry and baseline characteristics. Am J Epidemiol. 2018;187:1829-1839

19. Sterns RH. Disorders of plasma sodium - causes, consequences, and correction. N Engl J Med. 2015;372:55-65

20. George JC, Zafar W, Bucaloiu ID, et al. Risk factors and outcomes of rapid correction of severe hyponatremia. Clin J Am Soc Nephrol. 2018;13:984-992

21. Waikar SS, Mount DB, Curhan GC. Mortality after hospitalization with mild, moderate, and severe hyponatremia. Am J Med. 2009;122:857-865

22. Cárdenas A, Solà E, Rodríguez E, CANONIC study investigators of the EASL-CLIF Consortium, et al. Hyponatremia influences the outcome of patients with acute-on-chronic liver failure: an analysis of the CANONIC study. Crit Care. 2014;18:700

23. Krummel T, Prinz E, Metten MA, et al. Prognosis of patients with severe hyponatraemia is related not only to hyponatraemia but also to comorbidities and to medical management: results of an observational retrospective study. BMC Nephrol. 2016;17:159

24. Heuman DM, Abou-Assi SG, Habib A, et al. Persistent ascites and low serum sodium identify patients with cirrhosis and low MELD scores who are at high risk for early death. Hepatology. 2004;40:802-810

25. European Association for the Study of the Liver (2018) Electronic address: easloffice@easloffice.eu; European Association for the Study of the Liver EASL clinical practice guidelines for the management of patients with decompensated cirrhosis. J Hepatol 69:406-60.

26. John S, Thuluvath PJ. Hyponatremia in cirrhosis: pathophysiology and management. World J Gastroenterol. 2015;21:3197-3205

27. Ahluwalia V, Heuman DM, Feldman G, et al. Correction of hyponatraemia improves cognition, quality of life, and brain oedema in cirrhosis. J Hepatol. 2015;62:75-82

28. Garrahy A, Cuesta M, Murphy B, et al. Active management of severe hyponatraemia is associated with improved mortality. Eur J Endocrinol. 2021;184:9-17

29. Hernaez R, Solà E, Moreau R, Ginès P. Acute-on-chronic liver failure: an update. Gut. 2017;66(3):541-553

30. John S, Thuluvath PJ. Hyponatremia in cirrhosis: pathophysiology and management. World J Gastroenterol. 2015;21(11):3197-3205

Publisher's Note Springer Nature remains neutral with regard to jurisdictional claims in published maps and institutional affiliations. 


\section{Authors and Affiliations}

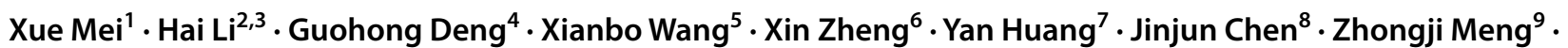
Yanhang Gao ${ }^{10}$. Feng $\mathrm{Liu}^{11}$ - Xiaobo $\mathrm{Lu}^{12} \cdot \mathrm{Yu} \mathrm{Shi}^{13,14,15}$. Yubao Zheng ${ }^{16}$. Huadong Yan ${ }^{17}$. Weituo Zhang ${ }^{18}$. Liang Qiao ${ }^{2,3}$. Wenyi Gu ${ }^{2,3}$. Yan Zhang ${ }^{2,3}$. Xiaomei Xiang ${ }^{4}$. Yi Zhou ${ }^{4}$. Shuning Sun ${ }^{4}$. Yixin $\mathrm{Hou}^{5}$. Qun Zhang ${ }^{5}$.

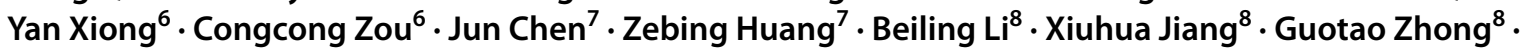

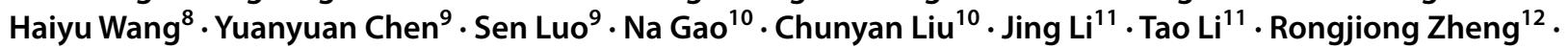
Xinyi Zhou ${ }^{12}$. Haotang Ren ${ }^{13,14,15}$. Wei Yuan ${ }^{1}$. Zhiping Qian ${ }^{1}$ (1)

1 Department of Liver Intensive Care Unit, Shanghai Public Health Clinical Centre (Fudan University), 2901 Cao Lang Road, Jinshan District, Shanghai 201508, China

2 Department of Gastroenterology, Ren Ji Hospital, School of Medicine, Shanghai Jiao Tong University, Shanghai, China

3 Shanghai Institute of Digestive Disease, Key Laboratory of Gastroenterology and Hepatology, Chinese Ministry of Health (Shanghai Jiao Tong University), Shanghai, China

4 Department of Infectious Diseases, Southwest Hospital, Third Military Medical University (Army Medical University), Chongqing, China

5 Center of Integrative Medicine, Beijing Ditan Hospital, Capital Medical University, Beijing, China

6 Department of Infectious Diseases, Institute of Infection and Immunology, Union Hospital, Tongji Medical College, Huazhong University of Science and Technology, Wuhan, China

7 Department of Infectious Diseases, Hunan Key Laboratory of Viral Hepatitis, Xiangya Hospital, Central South University, Changsha, China

8 Hepatology Unit, Department of Infectious Diseases, Nanfang Hospital, Southern Medical University, Guangzhou, China
9 Department of Infectious Diseases, Hubei Clinical Research Center for Precise Diagnosis and Treatment of Liver Cancer, Taihe Hospital, Hubei University of Medicine, Shiyan, China

10 Department of Hepatology, The First Hospital of Jilin University, Changchun, China

11 Department of Infectious Diseases and Hepatology, The Second Hospital of Shandong University, Jinan, China

12 Infectious Disease Center, First Affiliated Hospital of Xinjiang Medical University, Urumqi, China

13 The State Key Laboratory for Diagnosis and Treatment of Infectious Diseases, First Affiliated Hospital of School of Medicine, Zhejiang University, Hangzhou, China

14 Collaborative Innovation Center for Diagnosis and Treatment of Infectious Disease, Hangzhou, China

15 National Clinical Research Center of Infectious Disease, Hangzhou, China

16 Department of Infectious Diseases, Third Affiliated Hospital, Sun Yat-Sen University, Guangzhou, China

17 Department of Hepatology, Number 2 Hospital, Ningbo, China

18 Clinical Research Institute, School of Medicine, Shanghai Jiao Tong University, Shanghai, China 